\title{
Effect of First Order Auto-Regressive Model on the Power Function of Average Control Charts under Non-Normal Population
}

\author{
Uttama Mishra $^{1 *}$, J R Singh ${ }^{2}$ \\ ${ }^{1}$ Shri Vaishnav Vidyapeeth Vishwavidyalaya, India \\ ${ }^{2}$ Vikram University, Ujjain, India \\ *Corresponding Author: uttamamishra@gmail.com
}

Received: $20^{\text {th }}$ October 2020 / Revised: $4^{\text {th }}$ December $2020 /$ Published: $15^{\text {th }}$ December 2020

CIAppstat-SL2020

\begin{abstract}
The effect of First Order Auto-Regressive model and non-normality on the power function of the control charts for mean with control limits is studied. The nonnormality is represented by the first four terms of an Edgeworth series. The nonnormality must be unimodel. We have seen that power of control charts for mean is robust to normality with auto-regression.
\end{abstract}

Keywords: First Order Auto-Regressive Model, Power Function, Average Control Charts, Non-Normal Population.

\section{Introduction:}

Autoregressive processes employed under the rule that precedent values affect the existing values, which makes the statistical methods very much acknowledged for investigating nature, financial aspects, and other time-varying methods. Autoregressive models utilize a mixture of precedent values of the variable though multiple regression models estimate a variable utilizing a linear combination of forecasters. An AR (1) autoregressive process is the first order process, implying that the present value depends on the immediately preceding value. Control charts are very vital tools for quality monitoring and control with their long history of successful implementations and many practical applications. Constructions of control charts are based on the assumption that observations are independent and normally distributed. However, the assumption of independence of observations of quality characteristics is questionable; an autocorrelation among the observations becomes an inherent 
characteristic in quality control data, which leads to the erroneous conclusions about the state of control of the quality characteristics. Therefore, attention must be paid towards verifying the assumption of independence of data for constructing control chart while monitoring the quality characteristics.

Samanta and Bhattacherjee (2001) investigated the performance of control chart in the presence of auto-correlated data. Alwan (1992) suggested to widen the control limits of a control charts as a remedial method to deal with the auto-correlated data. Vasilopolousand Stamboulis (1978) constructed the control charts with the modified control limits width to processes described by first and second order auto-regressive model. Moreover, several authors have studied the control charts for correlated data under non-normal population. Naiki et al. (2013a, b) presented the economic statistical design of the variable sampling interval (VSI) $\bar{X}$ control charts for correlated and non-normal data. Naiki et al. (2013c) studied the economic design of VSI $\bar{X}$ control charts in the presence of multiple assignable causes with non-normal and correlated data.

\section{Power Function of the Average Control Chart under AR(1) Model for Non-normal Population:}

Consider the equation expressing AR (1) process given by the following model:

$$
x_{t}=\mu+\xi_{t}, t=1,2, \ldots n
$$

where $x_{t}$ is the response at time t, $\mu$ is a population mean (constant), $\xi_{t}$ is a stationary time series with zero mean and standard deviation $\sigma$. Suppose that a correlation test revealed the presence of data dependence and the identification technique suggested autoregressive model of order one AR (1) say, then $\xi_{t}$ can be expressed as:

$$
\xi_{t}=\rho_{1} \xi_{t-1}+\varepsilon_{t}, t=1,2,3, \ldots, n
$$

where

(i) $\varepsilon_{t} \sim N\left(0, \sigma_{\varepsilon}^{2}\right)$,

(ii) $\operatorname{Cov}\left(\varepsilon_{t}, \varepsilon_{\tau}\right)= \begin{cases}\sigma_{\varepsilon}^{2}, & t=\tau \\ 0, & t \neq \tau,\end{cases}$

When the correlation is present in the data we have for the distribution of sample mean $\bar{x}$, its mean and variance is as follows

$E(\bar{x})=\mu$,

$V(\bar{x})=\frac{\sigma^{2}}{n} \lambda\left(\alpha_{1}, n\right)=\frac{\sigma^{2}}{n} T^{2}$,

where $T^{2}=\lambda\left(\alpha_{1}, n\right)$. 
Effect of First Order Auto-Regressive Model on the Power Function of Average Control Charts under Non-Normal Population

For non-normal population, the density function is given by the first four terms of an Edgeworth series:

$$
f(x)=\frac{1}{\sigma}\left\{\phi\left(\frac{x-\mu}{\sigma}\right)-\frac{\lambda_{3}}{6} \phi^{(3)}\left(\frac{x-\mu}{\sigma}\right)+\frac{\lambda_{4}}{24} \phi^{(4)}\left(\frac{x-\mu}{\sigma}\right)+\frac{\lambda_{3}^{2}}{72} \phi^{(6)}\left(\frac{x-\mu}{\sigma}\right)\right\}
$$

The distribution of the sample mean is given by Singh (1983), as:

$g(\bar{x})=\frac{\sqrt{n}}{\sigma}\left\{\phi\left(\frac{\bar{x}-\mu}{T \sigma / \sqrt{n}}\right)-\frac{\lambda_{3} T}{6 \sqrt{n}} \phi^{(3)}\left(\frac{\bar{x}-\mu}{T \sigma / \sqrt{n}}\right)+\frac{\lambda_{4} T^{2}}{24 n} \phi^{(4)}\left(\frac{\bar{x}-\mu}{T \sigma / \sqrt{n}}\right)+\right.$

$\left.\frac{\lambda_{3}^{2} T^{2}}{72 n} \phi^{(6)}\left(\frac{\bar{x}-\mu}{T \sigma / \sqrt{n}}\right)\right\}$.

Now integrating Equation (7) after replacing $\mu$ by $\mu^{\prime}$, we have:

$\gamma(\bar{x})=\left\{\Phi\left(\frac{\bar{x}-\mu^{\prime}}{T \sigma / \sqrt{n}}\right)-\frac{\lambda_{3} T}{6 \sqrt{n}} \phi^{(2)}\left(\frac{\bar{x}-\mu^{\prime}}{T \sigma / \sqrt{n}}\right)+\frac{\lambda_{4} T^{2}}{24 n} \phi^{(3)}\left(\frac{\bar{x}-\mu^{\prime}}{T \sigma / \sqrt{n}}\right)+\frac{\lambda_{3}^{2} T^{2}}{72 n} \phi^{(5)}\left(\frac{\bar{x}-\mu^{\prime}}{T \sigma / \sqrt{n}}\right)\right\},(8)$

where

and

$$
\phi(x)=\frac{1}{\sqrt{2 \pi}} e^{-X^{2} / 2}
$$

If the samples of size $n$ are taken from the population $N\left(\mu^{\prime}, \sigma^{2} / n\right)$ and the value of the mean is plotted with the control limits $\mu \pm 3 \sigma / \sqrt{n}$, then the power of detecting the change of process is given by the following formula:

$$
P_{\bar{X}}=P_{r}\{\bar{X} \geq \mu+3 \sigma / \sqrt{n}\}+P_{r}\{\bar{X} \leq \mu-3 \sigma / \sqrt{n}\}
$$

Standardizing the above equation (9),

$$
Z=\frac{\bar{x}-\mu^{\prime}}{T \sigma / \sqrt{n}}
$$

The power function for normal distribution is obtained by converting Equation (9) into the standardized form, we have:

$$
\begin{aligned}
& P_{\bar{X}}=\left\{P_{r}\left(Z \geq\left(\mu-\mu^{\prime}\right) \sqrt{\frac{n}{T^{2} \sigma^{2}}}+\frac{3}{T}\right)+P_{r}\left(Z \leq\left(\mu-\mu^{\prime}\right) \sqrt{\frac{n}{T^{2} \sigma^{2}}}-\frac{3}{T}\right)\right\}, \\
&(2.9) \\
& P_{\bar{X}}=\left\{P_{r}\left(Z \geq \frac{-d \sqrt{n}}{T}+\frac{3}{T}\right)+P_{r}\left(Z \leq \frac{-d \sqrt{n}}{T}-\frac{3}{T}\right)\right\}, \\
&(2.10) \\
& P_{\bar{X}}=\left\{P_{r}\left(Z \leq \frac{1}{T}(d \sqrt{n}-3)\right)+P_{r}\left(Z \leq \frac{1}{T}(-d \sqrt{n}-3)\right)\right\}, \\
&(2.11) \\
& P_{\bar{X}}=\left\{\Phi\left(\frac{1}{T}(d \sqrt{n}-3)\right)+\Phi\left(\frac{1}{T}(-d \sqrt{n}-3)\right)\right\}, \\
& \text { whered }=\frac{\mu-\mu^{\prime}}{\sigma} .
\end{aligned}
$$

The Power function of the average control chart when the underlying population is non-normal is obtained by putting above value of the Equation (11) in the Equation (8): 


$$
\begin{aligned}
& P_{\bar{X}}=\left\{\Phi\left(\frac{1}{T}(d \sqrt{n}-3)\right)+\Phi\left(\frac{1}{T}(-d \sqrt{n}-3)\right)\right\}-\frac{\lambda_{3} T}{6 \sqrt{n}}\left\{\phi^{(2)}\left(\frac{1}{T}(d \sqrt{n}-3)\right)+\right. \\
& \left.\phi^{(2)}\left(\frac{1}{T}(-d \sqrt{n}-3)\right)\right\}+\frac{\lambda_{4} T^{2}}{24 n}\left\{\phi^{(3)}\left(\frac{1}{T}(d \sqrt{n}-3)\right)+\phi^{(3)}\left(\frac{1}{T}(-d \sqrt{n}-3)\right)\right\}+ \\
& \frac{\lambda_{3}^{2} T^{2}}{72 n}\left\{\phi^{(5)}\left(\frac{1}{T}(d \sqrt{n}-3)\right)+\phi^{(5)}\left(\frac{1}{T}(-d \sqrt{n}-3)\right)\right\} .
\end{aligned}
$$

The values of the power curve are obtained by using the Equation (12) is given in Table 1 to Table 9 and its diagrammatical representation is given in Figure 1 to Figure 4.

\section{Numerical Illustration and Conclusion:}

For the purpose of illustrating the effect of AR(1) process on the power function of average control chart under non-normality, we have determined the values of power function for different values of $\alpha_{1}=0.5,0.8,-0.5$ and different combinations of $\left(\lambda_{3}, \lambda_{4}\right)=$ $(0,0),(0,1),(0,0.5),(0.5,0),(-0.5,-0.5),(0.5,0.5),(-0.5,0),(0,-0.5)$ presented in the Table 1 to Table 9 when $n=5,10,15$ using out the Equation (12). In order to give the visual presentation of the power function for different values of $\alpha_{1}$ to the independent case, curves has been drawn for different values of $\lambda_{3}$ and $\lambda_{4}$ and is shown in the Figure 1 to Figure 4 when $n=5$. Visual presentation shows the relationship between the change of process, $d$, and the power of detecting this change, $P_{\bar{X}}$, when $n=5$.

\begin{tabular}{|c|c|c|c|c|c|c|c|c|}
\hline \multicolumn{9}{|c|}{$\alpha_{1}=0.5$} \\
\hline $\begin{array}{c}\left(\lambda_{3}, \lambda_{4}\right) \\
\rightarrow\end{array}$ & \multirow[t]{2}{*}{$(0,0)$} & \multirow[t]{2}{*}{$(0,1.0)$} & \multirow[t]{2}{*}{$(0,0.5)$} & \multirow[t]{2}{*}{$(0.5,0)$} & \multirow{2}{*}{$\left(\begin{array}{l}-0.5, \\
-0.5\end{array}\right)$} & \multirow[t]{2}{*}{$(0.5,0.5)$} & \multirow[t]{2}{*}{$(-0.5,0)$} & \multirow[t]{2}{*}{$(0,-0.5)$} \\
\hline$d \downarrow$ & & & & & & & & \\
\hline 0.0 & 0.04430 & 0.05630 & 0.05030 & $\begin{array}{r}0.02685 \\
3\end{array}$ & 0.05661 & 0.03285 & 0.06261 & 0.03831 \\
\hline 0.3 & 0.06613 & 0.07550 & 0.07082 & $\begin{array}{r}0.05144 \\
6\end{array}$ & 0.07648 & 0.05613 & 0.08116 & 0.06145 \\
\hline 0.5 & 0.10642 & 0.11190 & 0.10916 & $\begin{array}{r}0.09700 \\
3\end{array}$ & 0.11261 & 0.09975 & 0.11535 & 0.10367 \\
\hline
\end{tabular}

Table- 1: Values of Power Function for Average Control Chart under non-normal population when $n=5$. 
Effect of First Order Auto-Regressive Model on the Power Function of Average Control Charts under Non-Normal Population

\begin{tabular}{|l|l|l|l|r|c|c|c|c|}
\hline 0.8 & 0.20907 & 0.20888 & 0.20897 & 3 & 0.20401 & 0.21231 & 0.20392 & 0.20917 \\
\hline 1.0 & 0.30450 & 0.30267 & 0.30358 & 9 & 0.29054 & 0.31635 & 0.28962 & 0.30542 \\
\hline 1.3 & 0.47515 & 0.47486 & 0.47501 & 3 & 0.45315 & 0.49671 & 0.45300 & 0.47530 \\
\hline 1.5 & 0.59383 & 0.59551 & 0.59467 & 8 & 0.57376 & 0.61604 & 0.57459 & 0.59300 \\
\hline 1.8 & 0.75399 & 0.75613 & 0.75506 & 2 & 0.74694 & 0.76755 & 0.74800 & 0.75293 \\
\hline 2.0 & 0.83816 & 0.83828 & 0.83822 & 3 & 0.84165 & 0.84247 & 0.84171 & 0.83810 \\
\hline 2.5 & 0.95876 & 0.95302 & 0.95589 & 8 & 0.97297 & 0.94334 & 0.96601 & 0.96162 \\
\hline 3.0 & 0.99354 & 0.98921 & 0.99137 & 3 & 1.00000 & 0.95805 & 0.97068 & 0.99571 \\
\hline
\end{tabular}

Table- 2: Values of Power Function for Average Control Chart under non-normal population when $n=10$.

\begin{tabular}{|c|c|c|c|c|c|c|c|c|}
\hline \multicolumn{9}{|c|}{$\alpha_{1}=0.5$} \\
\hline $\begin{array}{c}\left(\lambda_{3}, \lambda_{4}\right) \\
\rightarrow\end{array}$ & \multirow[t]{2}{*}{$(0,0)$} & \multirow[t]{2}{*}{$(0,1.0)$} & \multirow[t]{2}{*}{$(0,0.5)$} & \multirow[t]{2}{*}{$(0.5,0)$} & \multirow{2}{*}{$\left(\begin{array}{l}-0.5, \\
-0.5\end{array}\right)$} & \multirow[t]{2}{*}{$(0.5,0.5)$} & \multirow[t]{2}{*}{$(-0.5,0)$} & \multirow[t]{2}{*}{$(0,-0.5)$} \\
\hline$d \downarrow$ & & & & & & & & \\
\hline \multirow{2}{*}{0.0} & & & & 0.0482 & & & & \\
\hline & 0.06283 & 0.06985 & 0.06634 & 4 & 0.07432 & 0.05175 & 0.07782 & 0.05932 \\
\hline \multirow{2}{*}{0.3} & & & & 0.0998 & & & & \\
\hline & 0.10884 & 0.11299 & 0.11092 & 5 & 0.11556 & 0.10193 & 0.11764 & 0.10677 \\
\hline \multirow{2}{*}{0.5} & & & & 0.1917 & & & & \\
\hline & 0.19171 & 0.19267 & 0.19219 & 4 & 0.19030 & 0.19221 & 0.19078 & 0.19124 \\
\hline \multirow{2}{*}{0.8} & & & & 0.3995 & & & & \\
\hline & 0.38561 & 0.38495 & 0.38528 & 0 & 0.37111 & 0.39917 & 0.37078 & 0.38594 \\
\hline \multirow{2}{*}{1.0} & & & & 0.5569 & & & & \\
\hline & 0.54015 & 0.54073 & 0.54044 & 4 & 0.52356 & 0.55723 & 0.52385 & 0.53986 \\
\hline \multirow{2}{*}{1.3} & & & & 0.7634 & & & & \\
\hline & 0.75457 & 0.75583 & 0.75520 & 8 & 0.74884 & 0.76411 & 0.74947 & 0.75395 \\
\hline \multirow{2}{*}{1.5} & & & & 0.8608 & & & & \\
\hline & 0.86018 & 0.85975 & 0.85997 & 2 & 0.86424 & 0.86060 & 0.86402 & 0.86040 \\
\hline
\end{tabular}




\begin{tabular}{|c|c|c|c|r|c|c|c|c|}
\hline 1.8 & 0.95249 & 0.94928 & 0.95088 & 4 & 0.96078 & 0.94254 & 0.95918 & 0.95409 \\
\hline 2.0 & 0.98038 & 0.97692 & 0.97865 & 4 & 0.98953 & 0.97291 & 0.98057 & 0.98211 \\
\hline 2.5 & 0.99883 & 0.99776 & 0.99829 & 7 & 1.00064 & 0.99684 & 1.00000 & 0.99936 \\
\hline 3.0 & 0.99997 & 0.99989 & 0.99993 & 4 & 1.00004 & 0.99990 & 1.00000 & 1.00000 \\
\hline
\end{tabular}

Table- 3: Values of Power Function for Average Control Chart under non-normal population when $n=15$.

\begin{tabular}{|c|c|c|c|c|c|c|c|c|}
\hline \multicolumn{9}{|c|}{$\alpha_{1}=0.5$} \\
\hline$\left(\lambda_{3}, \lambda_{4}\right)$ & \multirow{3}{*}{$(0,0)$} & \multirow{3}{*}{$(0,1.0)$} & \multirow{3}{*}{$(0,0.5)$} & \multirow{3}{*}{$(0.5,0)$} & \multirow{3}{*}{$\left(\begin{array}{l}-0.5, \\
-0.5\end{array}\right)$} & \multirow{3}{*}{$(0.5,0.5)$} & \multirow{3}{*}{$(-0.5,0)$} & \multirow{3}{*}{$(0,-0.5)$} \\
\hline$\rightarrow$ & & & & & & & & \\
\hline$d \downarrow$ & & & & & & & & \\
\hline 0.0 & 0.06959 & 0.07445 & 0.07202 & 0.05717 & 0.07983 & 0.05960 & 0.08226 & 0.06716 \\
\hline 0.3 & 0.13903 & 0.14117 & 0.14010 & 0.13387 & 0.14278 & 0.13494 & 0.14385 & 0.13796 \\
\hline 0.5 & 0.26144 & 0.26134 & 0.26139 & 0.26649 & 0.25562 & 0.26644 & 0.25557 & 0.26149 \\
\hline 0.8 & 0.52384 & 0.52418 & 0.52401 & 0.53787 & 0.50985 & 0.53803 & 0.51002 & 0.52367 \\
\hline 1.0 & 0.70128 & 0.70232 & 0.70180 & 0.71118 & 0.69293 & 0.71170 & 0.69345 & 0.70076 \\
\hline 1.3 & 0.89080 & 0.88991 & 0.89036 & 0.88874 & 0.89605 & 0.88829 & 0.89560 & 0.89125 \\
\hline 1.5 & 0.95537 & 0.95308 & 0.95422 & 0.94825 & 0.96205 & 0.94710 & 0.96090 & 0.95652 \\
\hline 1.8 & 0.99185 & 0.98991 & 0.99088 & 0.98839 & 0.99692 & 0.98742 & 0.99595 & 0.99282 \\
\hline 2.0 & 0.99795 & 0.99693 & 0.99744 & 0.99628 & 1.00012 & 0.99577 & 0.99962 & 0.99846 \\
\hline 2.5 & 0.99997 & 0.99992 & 0.99995 & 0.99988 & 1.00003 & 0.99985 & 1.00000 & 1.00000 \\
\hline 3.0 & 1.00000 & 1.00000 & 1.00000 & 1.00000 & 1.00000 & 1.00000 & 1.00000 & 1.00000 \\
\hline
\end{tabular}

Table- 4: Values of Power Function for Average Control Chart under non-normal population when $n=5$.

\begin{tabular}{|c|c|c|c|c|c|c|c|c|}
\hline \multicolumn{9}{|c|}{$\alpha_{1}=0.8$} \\
\hline $\begin{array}{c}\left(\lambda_{3}, \lambda_{4}\right) \\
\rightarrow\end{array}$ & \multirow[t]{2}{*}{$(0,0)$} & \multirow[t]{2}{*}{$(0,1.0)$} & \multirow[t]{2}{*}{$(0,0.5)$} & \multirow[t]{2}{*}{$(0.5,0)$} & \multirow{2}{*}{$\left(\begin{array}{l}-0.5, \\
-0.5\end{array}\right)$} & \multirow[t]{2}{*}{$(0.5,0.5)$} & \multirow[t]{2}{*}{$(-0.5,0)$} & \multirow[t]{2}{*}{$(0,-0.5)$} \\
\hline$d \downarrow$ & & & & & & & & \\
\hline 0.0 & 0.11492 & 0.13120 & 0.12306 & 0.09064 & 0.13103 & 0.09878 & 0.13917 & 0.10678 \\
\hline 0.3 & 0.13735 & 0.15066 & 0.14401 & 0.11834 & 0.14898 & 0.12500 & 0.15564 & 0.13070 \\
\hline 0.5 & 0.17658 & 0.18565 & 0.18111 & 0.16607 & 0.18083 & 0.17061 & 0.18536 & 0.17204 \\
\hline 0.8 & 0.26817 & 0.27126 & 0.26972 & 0.27409 & 0.25777 & 0.27563 & 0.25931 & 0.26663 \\
\hline
\end{tabular}


Effect of First Order Auto-Regressive Model on the Power Function of Average Control Charts under Non-Normal Population

\begin{tabular}{|l|l|l|l|l|l|l|l|l|}
\hline 1.0 & 0.34702 & 0.34822 & 0.34762 & 0.36328 & 0.32732 & 0.36388 & 0.32792 & 0.34642 \\
\hline 1.3 & 0.48144 & 0.48347 & 0.48245 & 0.50748 & 0.45402 & 0.50849 & 0.45503 & 0.48043 \\
\hline 1.5 & 0.57423 & 0.57790 & 0.57606 & 0.60137 & 0.54801 & 0.60321 & 0.54985 & 0.57239 \\
\hline 1.8 & 0.70502 & 0.70962 & 0.70732 & 0.72625 & 0.68998 & 0.72855 & 0.69228 & 0.70272 \\
\hline 2.0 & 0.78046 & 0.78354 & 0.78200 & 0.79448 & 0.77646 & 0.79602 & 0.77800 & 0.77892 \\
\hline 2.5 & 0.91326 & 0.90777 & 0.91051 & 0.90774 & 0.92963 & 0.90098 & 0.92689 & 0.91601 \\
\hline 3.0 & 0.97433 & 0.96450 & 0.96942 & 0.95008 & 0.97872 & 0.96007 & 0.98870 & 0.97924 \\
\hline
\end{tabular}

Table- 5: Values of Power Function for Average Control Chart under non-normal population when $n=10$.

\begin{tabular}{|c|c|c|c|c|c|c|c|c|}
\hline \multicolumn{9}{|c|}{$\alpha_{1}=0.8$} \\
\hline $\begin{array}{c}\left(\lambda_{3}, \lambda_{4}\right) \\
\rightarrow\end{array}$ & \multirow[t]{2}{*}{$(0,0)$} & \multirow[t]{2}{*}{$(0,1.0)$} & \multirow[t]{2}{*}{$(0,0.5)$} & \multirow[t]{2}{*}{$(0.5,0)$} & \multirow{2}{*}{$\left(\begin{array}{l}-0.5, \\
-0.5\end{array}\right)$} & \multirow[t]{2}{*}{$(0.5,0.5)$} & \multirow[t]{2}{*}{$(-0.5,0)$} & \multirow[t]{2}{*}{$(0,-0.5)$} \\
\hline$d \downarrow$ & & & & & & & & \\
\hline 0.0 & 0.19792 & 0.20460 & 0.20126 & 0.18304 & 0.20785 & 0.18638 & 0.21118 & 0.19459 \\
\hline 0.3 & 0.23441 & 0.24001 & 0.23721 & 0.22626 & 0.23780 & 0.22906 & 0.24060 & 0.23161 \\
\hline 0.5 & 0.29593 & 0.30042 & 0.29818 & 0.29750 & 0.28997 & 0.29975 & 0.29221 & 0.29369 \\
\hline 0.8 & 0.42886 & 0.43309 & 0.43097 & 0.44456 & 0.40997 & 0.44667 & 0.41208 & 0.42675 \\
\hline 1.0 & 0.53185 & 0.53681 & 0.53433 & 0.55226 & 0.51002 & 0.55474 & 0.51250 & 0.52937 \\
\hline 1.3 & 0.68438 & 0.68952 & 0.68695 & 0.70222 & 0.66972 & 0.70480 & 0.67230 & 0.68181 \\
\hline 1.5 & 0.77328 & 0.77673 & 0.77501 & 0.78472 & 0.76866 & 0.78645 & 0.77039 & 0.77155 \\
\hline 1.8 & 0.87612 & 0.87475 & 0.87543 & 0.87607 & 0.88578 & 0.87539 & 0.88509 & 0.87681 \\
\hline 2.0 & 0.92321 & 0.91857 & 0.92089 & 0.91623 & 0.93714 & 0.91203 & 0.93482 & 0.92553 \\
\hline 2.5 & 0.98237 & 0.97527 & 0.97882 & 0.97487 & 0.99678 & 0.97133 & 0.99323 & 0.98592 \\
\hline 3.0 & 0.99731 & 0.99380 & 0.99555 & 0.99399 & 1.00000 & 0.99223 & 1.00000 & 0.99907 \\
\hline
\end{tabular}

Table- 6: Values of Power Function for Average Control Chart under non-normal population whenn $=15$.

\begin{tabular}{|c|c|c|c|c|c|c|c|c|}
\hline \multicolumn{9}{|c|}{$\alpha_{1}=0.8$} \\
\hline $\begin{array}{c}\left(\lambda_{3}, \lambda_{4}\right) \\
\rightarrow\end{array}$ & \multirow{2}{*}{$(0,0)$} & \multirow{2}{*}{$(0,1.0)$} & \multirow[t]{2}{*}{$(0,0.5)$} & \multirow[t]{2}{*}{$(0.5,0)$} & \multirow{2}{*}{$\left(\begin{array}{l}-0.5 \\
-0.5\end{array}\right)$} & \multirow[t]{2}{*}{$(0.5,0.5)$} & \multirow{2}{*}{$(-0.5,0)$} & \multirow{2}{*}{$(0,-0.5)$} \\
\hline$d \downarrow$ & & & & & & & & \\
\hline 0.0 & 0.23667 & 0.23835 & 0.00000 & 0.22710 & 0.24273 & 0.22878 & 0.24441 & 0.23500 \\
\hline 0.3 & 0.28454 & 0.28618 & 0.00119 & 0.28194 & 0.28361 & 0.28358 & 0.28525 & 0.28291 \\
\hline 0.5 & 0.36318 & 0.36496 & 0.03892 & 0.36969 & 0.35332 & 0.37147 & 0.35510 & 0.36140 \\
\hline 0.8 & 0.52355 & 0.52596 & 0.56489 & 0.53984 & 0.50554 & 0.54225 & 0.50795 & 0.52114 \\
\hline
\end{tabular}




\begin{tabular}{|l|l|l|l|l|l|l|l|l|}
\hline 1.0 & 0.63806 & 0.64063 & 0.92622 & 0.65432 & 0.62259 & 0.65689 & 0.62516 & 0.63549 \\
\hline 1.3 & 0.78968 & 0.79109 & 0.99961 & 0.79754 & 0.78750 & 0.79895 & 0.78891 & 0.78827 \\
\hline 1.5 & 0.86637 & 0.86621 & 1.00000 & 0.86676 & 0.87345 & 0.86660 & 0.87329 & 0.86653 \\
\hline 1.8 & 0.94143 & 0.93914 & 1.00000 & 0.93222 & 0.95334 & 0.92993 & 0.95105 & 0.94372 \\
\hline 2.0 & 0.96941 & 0.96655 & 1.00000 & 0.95449 & 0.98327 & 0.95854 & 0.98040 & 0.97227 \\
\hline 2.5 & 0.99580 & 0.99408 & 1.00000 & 0.99208 & 1.00000 & 0.99035 & 1.00000 & 0.99753 \\
\hline 3.0 & 0.99966 & 0.99927 & 1.00000 & 0.99875 & 1.00000 & 0.99835 & 1.00000 & 1.00000 \\
\hline
\end{tabular}

Table- 7: Values of Power Function for Average Control Chart under non-normal population when $n=5$.

\begin{tabular}{|c|c|c|c|c|c|c|c|c|}
\hline \multicolumn{9}{|c|}{$\alpha_{1}=-0.5$} \\
\hline $\begin{array}{c}\left(\lambda_{3}, \lambda_{4}\right) \\
\rightarrow\end{array}$ & \multirow[t]{2}{*}{$(0,0)$} & \multirow[t]{2}{*}{$(0,1.0)$} & \multirow[t]{2}{*}{$(0,0.5)$} & \multirow[t]{2}{*}{$(0.5,0)$} & \multirow[t]{2}{*}{$\left(\begin{array}{l}-0.5, \\
-0.5\end{array}\right)$} & \multirow[t]{2}{*}{$(0.5,0.5)$} & \multirow[t]{2}{*}{$(-0.5,0)$} & \multirow[t]{2}{*}{$(0,-0.5)$} \\
\hline$d \downarrow$ & & & & & & & & \\
\hline 0.0 & 0.00000 & 0.00001 & 0.00001 & 0.00013 & 0.00001 & 0.00000 & 0.00001 & 0.00000 \\
\hline 0.3 & 0.00018 & 0.00028 & 0.00023 & 0.00556 & 0.00032 & 0.00004 & 0.00037 & 0.00013 \\
\hline 0.5 & 0.00195 & 0.00241 & 0.00218 & 0.02354 & 0.00284 & 0.00109 & 0.00307 & 0.00171 \\
\hline 0.8 & 0.03160 & 0.03274 & 0.03217 & 0.10939 & 0.03529 & 0.02797 & 0.03586 & 0.03102 \\
\hline 1.0 & 0.12064 & 0.12095 & 0.12079 & 0.22663 & 0.12221 & 0.11888 & 0.12236 & 0.12048 \\
\hline 1.3 & 0.44321 & 0.44302 & 0.44312 & 0.47744 & 0.43382 & 0.45242 & 0.43372 & 0.44331 \\
\hline 1.5 & 0.70649 & 0.70696 & 0.70673 & 0.65133 & 0.70086 & 0.71312 & 0.70109 & 0.70626 \\
\hline 1.8 & 0.94204 & 0.94109 & 0.94157 & 0.84860 & 0.94669 & 0.93745 & 0.94621 & 0.94252 \\
\hline 2.0 & 0.98803 & 0.98701 & 0.98752 & 0.92427 & 0.99185 & 0.98462 & 0.99134 & 0.98854 \\
\hline 2.5 & 0.99996 & 0.99993 & 0.99995 & 0.99241 & 1.00001 & 0.99988 & 1.00000 & 0.99998 \\
\hline 3.0 & 1.00000 & 1.00000 & 1.00000 & 0.99963 & 1.00000 & 1.00000 & 1.00000 & 1.00000 \\
\hline
\end{tabular}

Table-8: Values of Power Function for Average Control Chart under non-normal population when $n=10$.

\begin{tabular}{|c|c|c|c|c|c|c|c|c|}
\hline \multicolumn{9}{|c|}{$\alpha_{1}=-0.5$} \\
\hline $\begin{array}{c}\left(\lambda_{3}, \lambda_{4}\right) \\
\rightarrow\end{array}$ & \multirow[t]{2}{*}{$(0,0)$} & \multirow[t]{2}{*}{$(0,1.0)$} & \multirow[t]{2}{*}{$(0,0.5)$} & \multirow[t]{2}{*}{$(0.5,0)$} & \multirow{2}{*}{$\left(\begin{array}{l}-0.5, \\
-0.5\end{array}\right)$} & \multirow[t]{2}{*}{$(0.5,0.5)$} & \multirow[t]{2}{*}{$(-0.5,0)$} & \multirow[t]{2}{*}{$(0,-0.5)$} \\
\hline$d \downarrow$ & & & & & & & & \\
\hline 0.0 & 0.00000 & 0.00000 & 0.00000 & $\begin{array}{r}0.0000 \\
0\end{array}$ & 0.00000 & 0.00000 & 0.00000 & 0.00000 \\
\hline 0.3 & 0.00042 & 0.00050 & 0.00046 & $\begin{array}{r}0.0001 \\
8\end{array}$ & 0.00063 & 0.00022 & 0.00068 & 0.00038 \\
\hline
\end{tabular}


Effect of First Order Auto-Regressive Model on the Power Function of Average Control Charts under Non-Normal Population

\begin{tabular}{|c|c|c|c|r|c|c|c|c|}
\hline 0.5 & 0.01048 & 0.01092 & 0.01070 & 5 & 0.01223 & 0.00877 & 0.01245 & 0.01026 \\
\hline 0.8 & 0.22213 & 0.22198 & 0.22206 & 5 & 0.22012 & 0.22397 & 0.22005 & 0.22221 \\
\hline 1.0 & 0.60412 & 0.60427 & 0.60420 & 3 & 0.59835 & 0.61011 & 0.59842 & 0.60405 \\
\hline 1.3 & 0.96467 & 0.96416 & 0.96442 & 6 & 0.96791 & 0.96168 & 0.96718 & 0.96492 \\
\hline 1.5 & 0.99772 & 0.99750 & 0.99761 & 1 & 0.99865 & 0.99680 & 0.99854 & 0.99783 \\
\hline 1.8 & 0.99999 & 0.99999 & 0.99999 & 8 & 1.00000 & 0.99998 & 1.00000 & 1.00000 \\
\hline 2.0 & 1.00000 & 1.00000 & 1.00000 & 0 & 1.00000 & 1.00000 & 1.00000 & 1.00000 \\
\hline 2.5 & 1.00000 & 1.00000 & 1.00000 & 0 & 1.00000 & 1.00000 & 1.00000 & 1.00000 \\
\hline 3.0 & 1.00000 & 1.00000 & 1.00000 & 0 & 1.00000 & 1.00000 & 1.00000 & 1.00000 \\
\hline
\end{tabular}

Table-9: Values of Power Function for Average Control Chart under non-normal population whenn $=15$.

\begin{tabular}{|c|c|c|c|c|c|c|c|c|}
\hline \multicolumn{9}{|c|}{$\alpha_{1}=-0.5$} \\
\hline $\begin{array}{c}\left(\lambda_{3}, \lambda_{4}\right) \\
\rightarrow\end{array}$ & \multirow[t]{2}{*}{$(0,0)$} & \multirow[t]{2}{*}{$(0,1.0)$} & \multirow[t]{2}{*}{$(0,0.5)$} & \multirow[t]{2}{*}{$(0.5,0)$} & \multirow{2}{*}{$\left(\begin{array}{l}-0.5, \\
-0.5\end{array}\right)$} & \multirow[t]{2}{*}{$(0.5,0.5)$} & \multirow[t]{2}{*}{$(-0.5,0)$} & \multirow[t]{2}{*}{$(0,-0.5)$} \\
\hline$d \downarrow$ & & & & & & & & \\
\hline 0.0 & 0.00000 & 0.00000 & 0.00000 & $\begin{array}{r}0.0000 \\
0\end{array}$ & 0.00000 & 0.00000 & 0.00000 & 0.00000 \\
\hline 0.3 & 0.00114 & 0.00124 & 0.00119 & $\begin{array}{r}0.0007 \\
4\end{array}$ & 0.00151 & 0.00078 & 0.00155 & 0.00109 \\
\hline 0.5 & 0.03876 & 0.03908 & 0.03892 & $\begin{array}{r}0.0364 \\
6\end{array}$ & 0.04091 & 0.03662 & 0.04107 & 0.03860 \\
\hline 0.8 & 0.56486 & 0.56492 & 0.56489 & $\begin{array}{r}0.5698 \\
7\end{array}$ & 0.55990 & 0.56990 & 0.55993 & 0.56483 \\
\hline 1.0 & 0.92633 & 0.92611 & 0.92622 & $\begin{array}{r}0.9244 \\
3\end{array}$ & 0.92853 & 0.92432 & 0.92842 & 0.92645 \\
\hline 1.3 & 0.99963 & 0.99959 & 0.99961 & $\begin{array}{r}0.9994 \\
4\end{array}$ & 0.99983 & 0.99942 & 0.99980 & 0.99966 \\
\hline
\end{tabular}


Uttama Mishra and J R Singh

\begin{tabular}{|l|l|l|l|r|c|c|c|c|}
\hline 1.5 & 1.00000 & 1.00000 & 1.00000 & 0 & 1.0000 & & & \\
\\
\hline 1.8 & 1.00000 & 1.00000 & 1.00000 & 0 & 1.00000 & 1.00000 & 1.00000 & 1.00000 \\
\hline 2.0 & 1.00000 & 1.00000 & 1.00000 & 0 & 1.00000 & 1.00000 & 1.00000 & 1.00000 \\
\hline 2.5 & 1.00000 & 1.00000 & 1.00000 & 0 & 1.00000 & 1.00000 & 1.00000 & 1.00000 \\
\hline 3.0 & 1.00000 & 1.00000 & 1.00000 & 0 & 1.00000 & 1.00000 & 1.00000 & 1.00000 \\
\hline
\end{tabular}

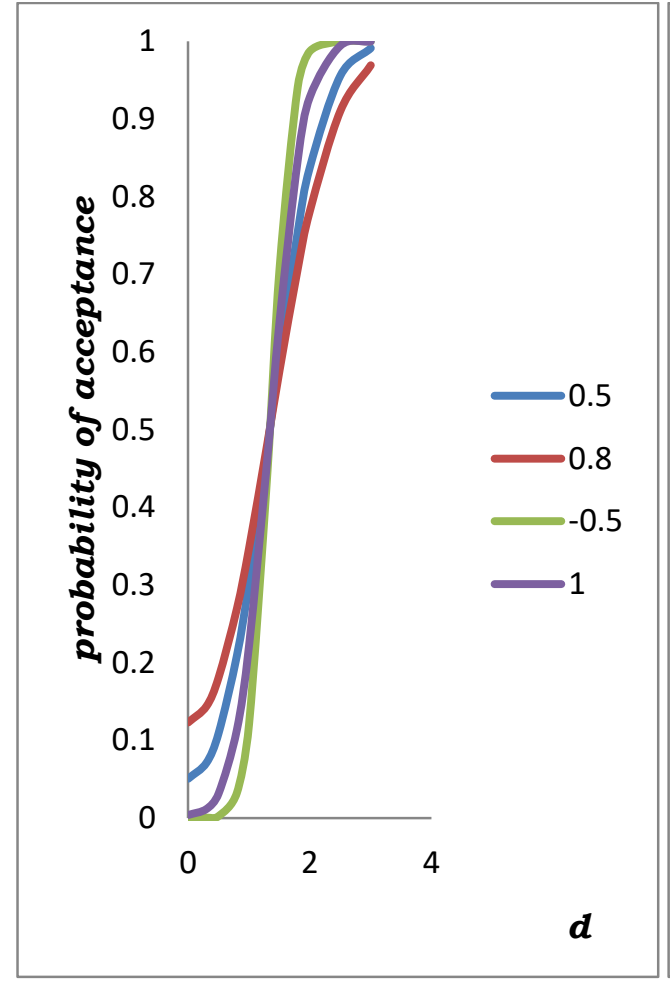

Figure 1: Power Curve for average control $\left(\lambda_{3}, \lambda_{4}\right)=(0,0.5)$

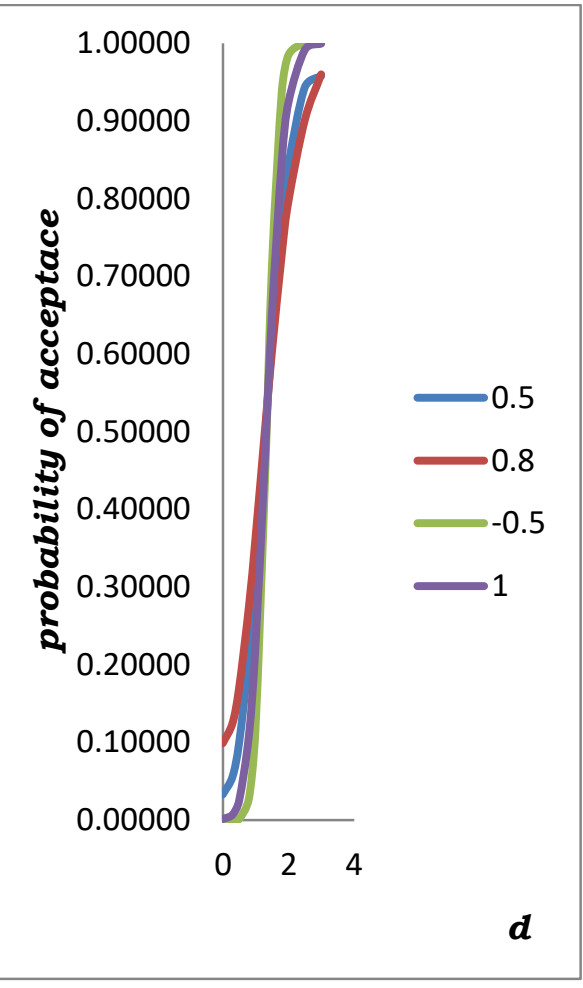

Figure2: Power Curve for when average control chart when $\left(\lambda_{3}, \lambda_{4}\right)=(0.5,0.5)$ 
Effect of First Order Auto-Regressive Model on the Power Function of Average Control Charts under Non-Normal Population

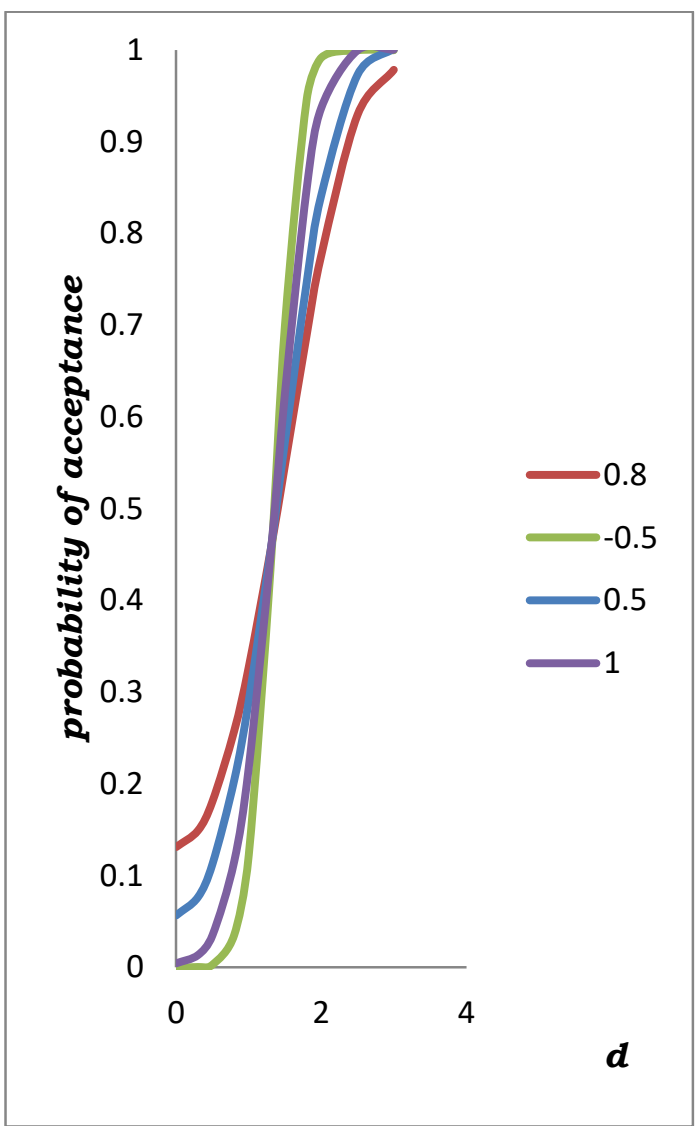

Figure 3: Power Curve for average control chart when $\left(\lambda_{3}, \lambda_{4}\right)=(0,0)$

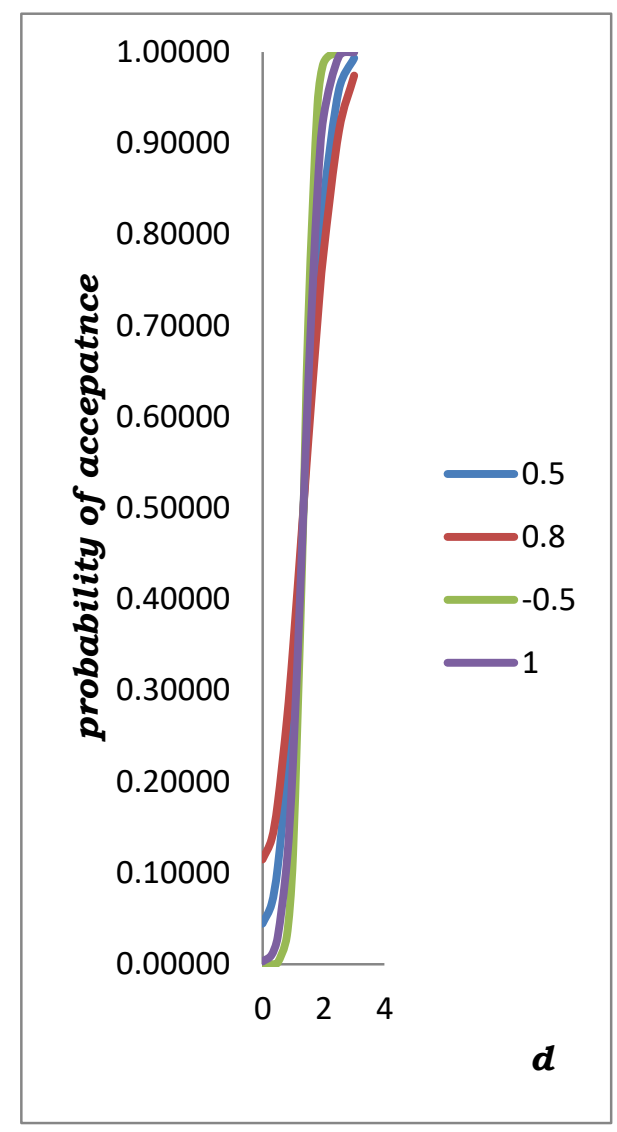

Figure 4: Power Curve for average control chart when $\left(\lambda_{3}, \lambda_{4}\right)=(-0.5,-0.5)$

From Table 1 to Table 9 it can be seen that the power of the control chart depends on the magnitude of the process change and different values of $\alpha_{1}, \lambda_{3}$ and $\lambda_{4}$ although, presence of autocorrelation results in the reduction of power when $d<1.5$ and reverse is the case when $d \geq 1.5$. Still when the autocorrelation is large enough to reduce the power under non-normality, the use of larger $n$ will result in an improvement. Conclusively, the practitioner who is fixed with the analysis and interpretation of auto-correlated data under non-normality, should first access the cause of the autocorrelation so that it will state what action(s) will be necessary. If a process generates data that are inherently auto-correlated, power function should be used to monitor any engineering control system that has been establish to minimize process variation about a target. 


\section{References}

1. Akhavan Naiki, S.T., Gazeneh, F. and Toosheghanian, M. (2013a). Economic Design of Variable Sampling Interval X-Bar Control Charts for Monitoring Correlated Non-Normal Samples, Communication in StatisticsTheory and Methods, 42 (18), 3339-3358.

2. Akhavan Naiki, S.T., Gazeneh, F. and Toosheghanian, M. (2013b). A Parameter-Tuned Genetic Algorithm for Economic Statistical Design for Variable Sampling Interval X-Bar Control Charts for Non-normal Correlated Samples, Communication in Statistics- Simulation and Computation, 43(5), 1212-1240.

3. Akhavan Naiki, S.T., Gazeneh, F. and Toosheghanian, M. (2013c). Economic Design of VSI $\bar{X}$ Control Charts with Correlated Non-normal Data under Multiple Assignable Causes, Journal of Statistical Computation and Simulation, 83(7), 1279-1300.

4. Alwan, L. C. (1992). Effect of Autocorrelation on Control Charts Performances, Communication in Statistics-Theory and Methods, 21, 10251049.

5. Samanta, B. and Bhattacherjee, A. (2001). An Investigation of Quality Control Charts for Auto-correlated data, Mineral Resources Engineering, 10(1), 53-69.

6. Singh, J.R. (1983). Further Developments of Control Charts and Acceptance Sampling plans, unpublished Ph.D. thesis, Dibrugarh University, Dibrugarh.

7. Vasilopolous, A.V. and Stamboulis, A. P. (1978). Modification of Control Charts Limits in the Presence of Data Correlation, Journal of Quality Technology, 10, 20-30. 